\title{
New aspects in polygroup theory
}

\author{
Andromeda Cristina Sonea
}

\begin{abstract}
The aim of this paper is to compute the commutativity degree in polygroup's theory, more exactly for the polygroup $P_{G}$ and for extension of polygroups by polygroups, obtaining boundaries for them. Also, we have analyzed the nilpotencitiy of $\mathcal{A}[\mathcal{B}]$, meaning the extension of polygroups $\mathcal{A}$ and $\mathcal{B}$.
\end{abstract}

\section{Introduction}

The polygroups theory represents a particular class from the hypergroup theory. This theory is detailed in the book of Davvaz, "Polygroup Theory and Related Systems" see [4]. We choose this class because it is similar to group theory and we founded a few similarities but and differences between these two theories.

Definition 1. A polygroup is a system $\varphi=\left\langle P, \cdot, e,^{-1}\right\rangle$, where $e \in P,{ }^{-1}$ is a unitary operation on $P$ and ". " $: P \times P \rightarrow \mathcal{P}^{*}(P)$. In the following, the next axioms hold for all $x, y, z \in P$ :

i) $(x \cdot y) \cdot z=x \cdot(y \cdot z)$;

ii) $e \cdot x=x \cdot e=x$;

iii) $x \in y \cdot z$, implies $y \in x \cdot z^{-1}$ and $z \in y^{-1} \cdot x$.

Key Words: polygroup, commutativity degree, extension of polygroups by polygroups, nilpotencity.

2010 Mathematics Subject Classification: Primary 46G05, 46L05; Secondary 47A30, 47B47.

Received: 27.12.2019

Accepted: 17.02 .2020 
Also, from the above axioms, it is obtaine:

$$
\begin{aligned}
e & \in x \cdot x^{-1} \cap x^{-1} \cdot x ; e^{-1}=e, \\
\left(x^{-1}\right)^{-1} & =x,(x \cdot y)^{-1}=y^{-1} \cdot x^{-1} .
\end{aligned}
$$

\section{Commutativity degree in polygroup theory}

The aim of this section is to compute the commutativity degree for polygroup $P_{G}$ and to find a connection between the results from group theory and from polygroup theory. This notion, was studied by Azam Hokmabadi, Fahimeh Mohammadzadeh and Elaheh Mohammadzade, see [7] presented in the $6^{\text {th }}$ International Group Theory Conference, 2014. In this paper, the definition of commutativity degree has a similar form, but we don't using the heart of a polygroup.

Definition 2. Let $\left\langle P, \cdot, e,^{-1}\right\rangle$ be a polygroup. The commutativity degree of polygroup $P$, notice by $d(P)$ has the next form:

$$
d(P)=\frac{\left|\left\{(a, b) \in P^{2} \mid a \cdot b=b \cdot a\right\}\right|}{|P|^{2}} .
$$

Remark 3. The set $\left\{(a, b) \in P^{2} \mid a \cdot b=b \cdot a\right\}$ is notice by $c(P)$.

Example 4. Let $P=\{e, a, b, c\}$ and let $\left\langle P, \cdot, e,^{-1}\right\rangle$ be a non-commutative polygroup, where". "is define thus

\begin{tabular}{l|l|l|l|l}
$\cdot$ & $e$ & $a$ & $b$ & $c$ \\
\hline$e$ & $e$ & $a$ & $b$ & $c$ \\
\hline$a$ & $a$ & $a$ & $P$ & $c$ \\
\hline$b$ & $b$ & $\{e, a, b\}$ & $b$ & $\{b, c\}$ \\
\hline$c$ & $c$ & $\{a, c\}$ & $c$ & $P$
\end{tabular}

In this case, the commutativity degree of polygroup $P$, is

$$
d(P)=\frac{10}{16}=\frac{5}{8}
$$

Proposition 5. Let $\left\langle P_{1}, \cdot, e_{1},{ }^{-1}>\right.$ and $\left\langle P_{2}, *, e_{2},{ }^{-1}>\right.$ be two polygroups. $P_{1} \times P_{2}$ equipped with the usual direct hyperproduct

$$
\begin{gathered}
" \circ ":\left(P_{1} \times P_{2}\right) \times\left(P_{1} \times P_{2}\right) \rightarrow P_{1} \times P_{2}, \\
\left(x_{1}, y_{1}\right) \circ\left(x_{2}, y_{2}\right)=\left\{(x, y) \mid x \in x_{1} \cdot x_{2}, y \in y_{1} * y_{2}\right\}
\end{gathered}
$$

becomes a polygroup. 
Proposition 6. Let $\left\langle P_{1}, \cdot, e_{1},{ }^{-1}>\right.$ and $\left\langle P_{2}, *, e_{2},{ }^{-1}\right\rangle$ be two polygroups. The next relation holds

$$
d\left(P_{1} \times P_{2}\right)=d\left(P_{1}\right) d\left(P_{2}\right) .
$$

Proof. The amount

$$
\frac{\left|\left\{\left(x_{1}, y_{1}\right) \times\left(x_{2}, y_{2}\right) \in\left(P_{1} \times P_{2}\right)^{2} \mid\left(x_{1}, y_{1}\right) \circ\left(x_{2}, y_{2}\right)=\left(x_{2}, y_{2}\right) \circ\left(x_{1}, y_{1}\right)\right\}\right|}{\left|P_{1} \times P_{2}\right|^{2}} .
$$

represents the commutativity degree of $P_{1} \times P_{2}$. So, the expression

$$
\left(x_{1}, y_{1}\right) \circ\left(x_{2}, y_{2}\right)=\left(x_{2}, y_{2}\right) \circ\left(x_{1}, y_{1}\right)
$$

is equivalent with

$$
\begin{aligned}
\{(x, y) & \left.\in P_{1} \times P_{2} \mid x \in x_{1} \cdot x_{2}=x_{2} \cdot x_{1}, y \in y_{1} * y_{2}=y_{2} * y_{1}\right\} \\
& =\left\{x \in P_{1} \mid x \in x_{1} \cdot x_{2}=x_{2} \cdot x_{1}\right\}\left\{y \in P_{2} \mid y \in y_{1} * y_{2}=y_{2} * y_{1}\right\} \\
& =c\left(P_{1}\right) c\left(P_{2}\right) . \\
P_{1} \times P_{2} & =\left\{(x, y) \mid x \in P_{1}, y \in P_{2}\right\}=\left\{x, x \in P_{1}\right\}\left\{y, y \in P_{2}\right\},
\end{aligned}
$$

follows that

$$
\left|P_{1} \times P_{2}\right|=\left|P_{1}\right|\left|P_{2}\right|
$$

Therefore,

$$
d\left(P_{1} \times P_{2}\right)=\frac{\left|c\left(P_{1} \times P_{2}\right)\right|}{\left|P_{1} \times P_{2}\right|^{2}}=\frac{\left|c\left(P_{1}\right)\right|\left|c\left(P_{2}\right)\right|}{\left|P_{1} \times P_{2}\right|^{2}} .
$$

In conclusion,

$$
d\left(P_{1} \times P_{2}\right)=\frac{\left|c\left(P_{1}\right)\right|}{\left|P_{1}\right|^{2}} \frac{\left|c\left(P_{2}\right)\right|}{\left|P_{2}\right|^{2}}=d\left(P_{1}\right) d\left(P_{2}\right),
$$

Example 7. Let sets $P_{1}=\{0,1\}, P_{2}=\{e, a, b, c\}$ and let $\left\langle P_{1}, \cdot, e,^{-1}\right\rangle$, $<P_{2}, *, e^{\prime},{ }^{-1}>$ be two polygroups, where.$"$ ş $" *$ " are define thus:

\begin{tabular}{|l|l|l|}
\hline$\cdot$ & 0 & 1 \\
\hline 0 & 0 & 1 \\
\hline 1 & 1 & 0 \\
\hline
\end{tabular}

and

\begin{tabular}{|l|l|l|l|l|}
\hline$*$ & $e$ & $a$ & $b$ & $c$ \\
\hline$e$ & $e$ & $a$ & $b$ & $c$ \\
\hline$a$ & $a$ & $a$ & $P_{2}$ & $c$ \\
\hline$b$ & $b$ & $\{e, a, b\}$ & $b$ & $\{b, c\}$ \\
\hline$c$ & $c$ & $\{a, c\}$ & $c$ & $P_{2}$ \\
\hline
\end{tabular}


We notice

$$
\alpha_{i}^{j}=\left(x_{i}, y_{j}\right), i \in\{1,2\}, j \in\{1,2,3\},
$$

where $x_{i}$ and $y_{j}$, represents of component $i$ from $P_{1}$ and $y_{j}$ represents of component $j$ from $P_{2}$. The product polygroup $P_{1} \times P_{2}$ has the next form.

\begin{tabular}{|l|l|l|l|l|}
\hline$\circ$ & $\alpha_{1}^{1}$ & $\alpha_{1}^{2}$ & $\alpha_{1}^{3}$ & $\alpha_{1}^{4}$ \\
\hline$\alpha_{1}^{1}$ & $\alpha_{1}^{1}$ & $\alpha_{1}^{2}$ & $\alpha_{1}^{3}$ & $\alpha_{1}^{4}$ \\
\hline$\alpha_{1}^{2}$ & $\alpha_{1}^{2}$ & $\alpha_{1}^{2}$ & $\left\{\begin{array}{c}\alpha_{1}^{i}, \\
i=\overline{1,4}\end{array}\right\}$ & $\alpha_{1}^{4}$ \\
\hline$\alpha_{1}^{3}$ & $\alpha_{1}^{3}$ & $\left\{\begin{array}{c}\alpha_{1}^{i}, \\
i=\overline{1,3}\end{array}\right\}$ & $\alpha_{1}^{3}$ & $\left\{\alpha_{1}^{3}, \alpha_{1}^{4}\right\}$ \\
\hline$\alpha_{1}^{4}$ & $\alpha_{1}^{4}$ & $\left\{\alpha_{1}^{2}, \alpha_{1}^{4}\right\}$ & $\alpha_{1}^{4}$ & $\left\{\begin{array}{c}\alpha_{1}^{i}, \\
i=\overline{1,4}\end{array}\right\}$ \\
\hline$\alpha_{2}^{1}$ & $\alpha_{2}^{1}$ & $\alpha_{2}^{2}$ & $\alpha_{2}^{3}$ & $\alpha_{2}^{4}$ \\
\hline$\alpha_{2}^{2}$ & $\alpha_{2}^{2}$ & $\alpha_{2}^{2}$ & $\left\{\begin{array}{c}\alpha_{2}^{i}, \\
i=\overline{1,4}\end{array}\right\}$ & $\alpha_{2}^{4}$ \\
\hline$\alpha_{2}^{3}$ & $\alpha_{2}^{3}$ & $\left\{\begin{array}{c}\alpha_{2}^{i}, \\
i=\overline{1,3}\end{array}\right\}$ & $\alpha_{2}^{3}$ & $\left\{\alpha_{2}^{3}, \alpha_{2}^{4}\right\}$ \\
\hline$\alpha_{2}^{4}$ & $\alpha_{2}^{4}$ & $\left\{\alpha_{2}^{2}, \alpha_{2}^{4}\right\}$ & $\alpha_{2}^{4}$ & $\left\{\begin{array}{c}\alpha_{2}^{i}, \\
i=\overline{1,4}\end{array}\right\}$ \\
\hline
\end{tabular}

and

\begin{tabular}{|c|c|c|c|c|}
\hline$\circ$ & $\alpha_{2}^{1}$ & $\alpha_{2}^{2}$ & $\alpha_{2}^{3}$ & $\alpha_{2}^{4}$ \\
\hline$\alpha_{1}^{1}$ & $\alpha_{2}^{1}$ & $\alpha_{2}^{2}$ & $\alpha_{2}^{3}$ & $\alpha_{2}^{4}$ \\
\hline$\alpha_{1}^{2}$ & $\alpha_{2}^{2}$ & $\alpha_{2}^{2}$ & $\left\{\alpha_{2}^{i}, i=\overline{1,4}\right\}$ & $\alpha_{2}^{4}$ \\
\hline$\alpha_{1}^{3}$ & $\alpha_{2}^{3}$ & $\left\{\alpha_{2}^{i}, i=\overline{1,3}\right\}$ & $\alpha_{2}^{3}$ & $\left\{\alpha_{2}^{3}, \alpha_{2}^{4}\right\}$ \\
\hline$\alpha_{1}^{4}$ & $\alpha_{2}^{4}$ & $\left\{\alpha_{2}^{2}, \alpha_{2}^{4}\right\}$ & $\alpha_{2}^{4}$ & $\left\{\alpha_{2}^{i}, i=\overline{1,4}\right\}$ \\
\hline$\alpha_{2}^{1}$ & $\alpha_{1}^{1}$ & $\alpha_{1}^{2}$ & $\alpha_{1}^{3}$ & $\alpha_{1}^{4}$ \\
\hline$\alpha_{2}^{2}$ & $\alpha_{1}^{2}$ & $\alpha_{1}^{2}$ & $\left\{\alpha_{1}^{i}, i=\overline{1,4}\right\}$ & $\alpha_{1}^{4}$ \\
\hline$\alpha_{2}^{3}$ & $\alpha_{1}^{3}$ & $\left\{\alpha_{1}^{i}, i=\overline{1,3}\right\}$ & $\alpha_{1}^{3}$ & $\left\{\alpha_{1}^{3}, \alpha_{1}^{4}\right\}$ \\
\hline$\alpha_{2}^{4}$ & $\alpha_{1}^{4}$ & $\left\{\alpha_{1}^{2}, \alpha_{1}^{4}\right\}$ & $\alpha_{1}^{4}$ & $\left\{\alpha_{1}^{i}, i=\overline{1,4}\right\}$ \\
\hline
\end{tabular}

The commutativity degree is

$$
d\left(P_{1} \times P_{2}\right)=\frac{40}{64}=\frac{5}{8} \cdot 1=d\left(P_{1}\right) \cdot d\left(P_{2}\right) .
$$

Let $(G, \cdot)$ be a group and $P_{G}=G \cup\{a\}$, where $a \notin G$. It is define on $P_{G}$, 
the hyperoperation " ○" as follows

$$
\begin{aligned}
& (1) \quad: \quad a \circ a=e \\
& (2) \quad: \quad e \circ x=x \circ e=x, \forall x \in P_{G} \\
& (3) \quad: \quad a \circ x=x \circ a=x, \forall x \in P_{G} \backslash\{e, a\} \\
& (4) \quad: \quad x \circ y=x \cdot y, \forall(x, y) \in G^{2}, y \neq x^{-1} ; \\
& (5) \quad: \quad x \circ x^{-1}=\{e, a\}, \forall x \in P_{G} \backslash\{e, a\} .
\end{aligned}
$$

Proposition 8. If $G$ is a group, then $\left\langle P_{G}, \circ, e,{ }^{-1}\right\rangle$ is a polygroup.

Corolar 9. Let $(G, \cdot)$ be a group. The polygroup $P_{G}$ is nilpotent, if and only if $G$ is a nilpotent group.

Proposition 10. If $(G, \cdot)$ is a finit group, with $|G|=n, n \in \mathbb{N}^{*}$, then

$$
d\left(P_{G}\right)=\frac{n^{2} d(G)+2 n+1}{(n+1)^{2}} .
$$

Proof. We define, the commutativity degree of polygroup $P_{G}$ as follows

$$
d\left(P_{G}\right)=\frac{\left|\left\{(x, y) \in P_{G}^{2} \mid x \circ y=y \circ x\right\}\right|}{\left|P_{G}\right|^{2}} .
$$

Let

$$
\begin{aligned}
& A_{1}=\left\{(x, y) \in G^{2}, y \neq x^{-1}\right\}, A_{2}=\left\{(x, y) \in G^{2}, y=x^{-1}\right\} \\
& A_{3}=\{(a, y), y \in G\}, A_{4}=\{(x, a), x \in G, y=a\}, A_{5}=\{(a, a)\} .
\end{aligned}
$$

We observe that

$$
P_{G} \times P_{G}=A_{1} \cup A_{2} \cup A_{3} \cup A_{4} \cup A_{5},
$$

with

$$
A_{i} \cap A_{j}=\varnothing, \forall i \neq j .
$$

According to (5) and (6), the above expression, could be written thus

$$
\begin{aligned}
\left|\left\{(x, y) \in P_{G}^{2} \mid x \circ y=y \circ x\right\}\right| & =\sum_{i=1}^{5} \mid\left\{(x, y) \in A_{i}|x \circ y=y \circ x|\right. \\
& =n^{2} d(G)+n+n+1=n^{2} d(G)+2 n+1 .
\end{aligned}
$$

So,

$$
d\left(P_{G}\right)=\frac{n^{2} d(G)+2 n+1}{(n+1)^{2}} .
$$


Example 11. If $G=D_{3}$, then $P_{G}=G \cup a, a \notin D_{3}$. The commutativity degree of $G$, is $d(G)=\frac{1}{2}$.

\begin{tabular}{|l|l|l|l|l|l|l|l|}
\hline$\circ$ & $e$ & $\rho$ & $\rho^{2}$ & $\sigma$ & $\rho \sigma$ & $\rho^{2} \sigma$ & $a$ \\
\hline$e$ & $e$ & $\rho$ & $\rho^{2}$ & $\sigma$ & $\rho \sigma$ & $\rho^{2} \sigma$ & $a$ \\
\hline$\rho$ & $\rho$ & $\rho^{2}$ & $\{e, a\}$ & $\rho \sigma$ & $\rho^{2} \sigma$ & $\sigma$ & $\rho$ \\
\hline$\rho^{2}$ & $\rho^{2}$ & $\{e, a\}$ & $\rho$ & $\rho^{2} \sigma$ & $\sigma$ & $\rho \sigma$ & $\rho^{2}$ \\
\hline$\sigma$ & $\sigma$ & $\rho^{2} \sigma$ & $\rho \sigma$ & $\{e, a\}$ & $\rho^{2}$ & $\rho$ & $\sigma$ \\
\hline$\rho \sigma$ & $\rho \sigma$ & $\sigma$ & $\rho^{2} \sigma$ & $\rho$ & $\{e, a\}$ & $\rho^{2}$ & $\rho$ \\
\hline$\rho^{2} \sigma$ & $\rho^{2} \sigma$ & $\rho \sigma$ & $\sigma$ & $\rho^{2}$ & $\rho$ & $\{e, a\}$ & $\rho^{2} \sigma$ \\
\hline$a$ & $a$ & $\rho$ & $\rho^{2}$ & $\sigma$ & $\rho \sigma$ & $\rho^{2} \sigma$ & $e$ \\
\hline
\end{tabular}

$$
d\left(P_{G}\right)=\frac{31}{49}=\frac{6^{2} \cdot \frac{1}{2}+2 \cdot 6+1}{7^{2}} .
$$

Remark 12. 1. $d\left(P_{G}\right) \geq d(G)$, for all group $G$;

2. If $G$ is an abelian group, then $P_{G}$ is a commutative polygroup.

3. According to the above example, it is observed that there is a non commutative polygroup $P_{G}$ with commutativity degree more than $\frac{5}{8}$, what in group theory does not happend.

In what follows, we determine a bounded for polygroup $P_{G}$, which depends to $d(G)$.

Proposition 13. If $G$ is a group with $|G|=n$, then

$$
d(G) \leq d\left(P_{G}\right) \leq \frac{d(G)+3}{4} .
$$

Proof. Let $G$ be a group, with $|G|=n$. The first inequality is obvious, from Remark 12 and for second inequality, we make some elementary calculus and we obtain

$$
(d(G)-1)\left(3 n^{2}-2 n-1\right) \leq 0, \forall n \geq 1 .
$$

It is true, because $d(G) \in(0,1]$ and $3 n^{2}-2 n-1=(n-1)(3 n+1) \geq 0, \forall n \geq$ 1.

Proposition 14. $P_{G}$ is a commutative polygroup if and only if $d\left(P_{G}\right)>\frac{29}{32}$.

Proof. If $P_{G}$ is commutative polygroup, follows that $d\left(P_{G}\right)=1>\frac{29}{32}$.

Inverse, if $d\left(P_{G}\right)>\frac{29}{32}$, then

$$
\begin{aligned}
\frac{n^{2} d(G)+2 n+1}{(n+1)^{2}} & >\frac{29}{32}, \text { equivalent } \\
n^{2}(32 d(G)-29)+6 n+3 & >0, \text { for all } n \geq 2 .
\end{aligned}
$$


If $G$ is abelian group, then $d(G)=1$ and inequality is true.

If $G$ is a non abelian group, then $d(G)<\frac{5}{8}$, so

$$
n^{2}(32 d(G)-29)+6 n+3<-9 n^{2}+6 n+3<0 \text {, for all } n \geq 2 \text {. }
$$

In this situation, the inequality doesn't holds. $\frac{29}{32}$.

In conclusion, $P_{G}$ is a commutative polygroup if and only if $d\left(P_{G}\right)>$

\section{Extension of polygroups by polygroups}

The purpose of this section is to determine the commutativity degree of extension polygroups by polygroups and to find a connection with the commutativity degrees of the two polygroups which form the extension. Let $\mathcal{A}=<$ $\left.A, \cdot, e^{-1}\right\rangle$ and $\mathcal{B}=\left\langle B, \cdot, e,^{-1}\right\rangle$ be two polygroups whose elements have been renamed so that încât $A \cap B=\{e\}$. A new system $\mathcal{A}[\mathcal{B}]$ called the extension of $\mathcal{A}$ by $\mathcal{B}$ is formed in the following way:

$$
\mathcal{A}[\mathcal{B}]=\left\langle M, *, e,^{I}\right\rangle,
$$

where

$$
M=A \cup B, e^{I}=e, x^{I}=x^{-1}, e * x=x * e=x, \text { for all } x \in M ;
$$

and for all $x, y \in M \backslash\{e\}$.

$$
x * y= \begin{cases}x \cdot y & \text { if } x, y \in A \\ x & \text { if } x \in B, y \in A \\ y & \text { if } x \in A, y \in B \\ x \cdot y & \text { if } x, y \in B, y \neq x^{-1} \\ x \cdot y \cup A & \text { if } x, y \in B, y=x^{-1}\end{cases}
$$

\begin{tabular}{|c|c|c|c|c|c|c|c|c|c|}
\hline$*$ & $e$ & $a_{1}$ & $\ldots$ & $a_{n-1}$ & $b_{1}$ & $\ldots$ & $b_{i}$ & $\ldots$ & $b_{m-1}$ \\
\hline$e$ & $e$ & $a_{1}$ & $\ldots$ & $a_{n-1}$ & $b_{1}$ & $\ldots$ & $b_{i}$ & $\ldots$ & $b_{m-1}$ \\
\hline$a_{1}$ & $a_{1}$ & $a_{1} a_{1}$ & $\ldots$ & $a_{1} a_{n-1}$ & $b_{1}$ & $\ldots$ & $b_{i}$ & $\ldots$ & $b_{m-1}$ \\
\hline : & : & . & & . & : & & : & & : \\
\hline$\frac{\cdot}{a_{n-1}}$ & $\frac{\cdot}{a_{n-1}}$ & $a_{n-1} a_{1}$ & $\begin{array}{l}\ldots \\
\ldots\end{array}$ & $a_{n-1} a_{n-1}$ & $b_{1}$ & $\begin{array}{l}\ldots \\
\ldots\end{array}$ & $\dot{b_{i}}$ & $\begin{array}{l}\ldots \\
\ldots\end{array}$ & $b_{m-1}$ \\
\hline$b_{1}$ & $b_{1}$ & $b_{1}$ & $\ldots$ & $b_{1}$ & $b_{1} b_{1}$ & $\ldots$ & $b_{1} b_{i} \cup A$ & $\ldots$ & $b_{1} b_{m-1}$ \\
\hline : & . & . & $\ldots$ & . & & $\ldots$ & : & $\ldots$ & : \\
\hline$b_{i}$ & $b_{i}$ & $b_{i}$ & $\ldots$ & $b_{i}$ & $b_{i} b_{1} \cup A$ & $\ldots$ & $b_{i} b_{i}$ & $\ldots$ & $b_{i} b_{m-1}$ \\
\hline$\vdots$ & : & . & $\ldots$ & $\vdots$ & : & $\ldots$ & $\vdots$ & $\ldots$ & : \\
\hline$b_{m-1}$ & $b_{m-1}$ & $b_{m-1}$ & $\ldots$ & $b_{m-1}$ & $b_{m-1} b_{1}$ & $\ldots$ & $b_{m-1} b_{i}$ & $\ldots$ & $b_{m-1} b_{m-1}$ \\
\hline
\end{tabular}

In this case, $\mathcal{A}[\mathcal{B}]$ is a polygroup which is called the extension of $\mathcal{A}$ by $\mathcal{B}$.

We consider $A=\left\{e, a_{1}, a_{2}, \ldots, a_{n-1}\right\}$ şi $B=\left\{e, b_{1}, b_{2}, \ldots, b_{m-1}\right\}$, where $n$, $m \in \mathbb{N}^{*}$. We can represent the operation " * " through next table: 
Without loss generality, we suppose that $b_{i}=b_{1}^{-1}$. For each element $b_{j}$, it is exists unique $b_{k}$, such that $b_{j}=b_{k}^{-1}$ with $i, j, k \in \overline{1, m-1}$.

The commutativity degree of polygroup $\mathcal{A}[\mathcal{B}]$ it is define thus:

$$
d(\mathcal{A}[\mathcal{B}])=\frac{\left|\left\{(x, y) \in M^{2} \mid x * y=y * x\right\}\right|}{|M|^{2}} .
$$

Proposition 15. If $\mathcal{A}=\left\langle A, \cdot, e,^{-1}\right\rangle$ and $\mathcal{B}=\left\langle B, \cdot, e,^{-1}\right\rangle$ are two finite polygroups, where $A=\left\{e, a_{1}, a_{2}, \ldots, a_{n-1}\right\}$ şi $B=\left\{e, b_{1}, b_{2}, \ldots, b_{m-1}\right\}$, with $n$, $m \in \mathbb{N}^{*}$, then the commutativity degree of polygroup $\mathcal{A}[\mathcal{B}]$, is

$$
d(\mathcal{A}[\mathcal{B}])=\frac{n^{2} d(\mathcal{A})+m^{2} d(\mathcal{B})+2(n-1)(m-1)-1}{(n+m-1)^{2}} .
$$

Proof. Let sets

$$
\begin{aligned}
& A_{1}=\left\{(x, y) \in A^{2} \mid x * y=y * x\right\} \\
& A_{2}=\left\{(x, y) \in B^{2} \mid x * y=y * x\right\} \\
& A_{3}=\{(x, y) \in A \times B \mid x * y=y * x, x, y \neq e\} \\
& A_{4}=\{(x, y) \in B \times A \mid x * y=y * x, x, y \neq e\} .
\end{aligned}
$$

It is easy to observe that

$$
\begin{aligned}
A_{1} \cap A_{2} & =\{(e, e)\} \\
A_{i} \cap A_{j} & =\emptyset, \forall(i, j) \neq(1,2), i, j=\overline{1,4} .
\end{aligned}
$$

and

$$
\left\{(x, y) \in M^{2} \mid x * y=y * x\right\}=\bigcup_{i=1}^{4} A_{i} .
$$

In conclusion,

$$
d(\mathcal{A}[\mathcal{B}])=\frac{n^{2} d(\mathcal{A})+m^{2} d(\mathcal{B})+2(n-1)(m-1)-1}{(n+m-1)^{2}} .
$$

Example 16. Let $\mathcal{P}_{1}=\left\langle P_{1}, \cdot, e,^{-1}\right\rangle$ and $\mathcal{P}_{2}=\left\langle P_{2}, \cdot, e,,^{-1}\right\rangle$ be two polygroups, where $P_{1}=\{e, a, b, c\}$ and $P_{2}=\left\{e, a^{\prime}, b^{\prime}\right\}$ thus :

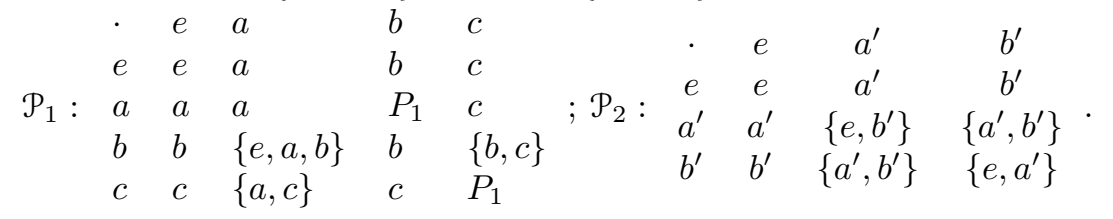


The extension of polygroup $\mathcal{P}_{1}$ by polygroup $\mathcal{P}_{2}, \mathcal{P}_{1}\left[\mathcal{P}_{2}\right]=\left\langle M, *, e,{ }^{I}\right\rangle$ it is represents as follows

$\begin{array}{ccccccc}* & e & a & b & c & a^{\prime} & b^{\prime} \\ e & e & a & b & c & a^{\prime} & b^{\prime} \\ a & a & a & P_{1} & c & a^{\prime} & b^{\prime} \\ b & b & \{e, a, b\} & b & \{b, c\} & a^{\prime} & b^{\prime} \\ c & c & \{a, c\} & c & P_{1} & a^{\prime} & b^{\prime} \\ a^{\prime} & a^{\prime} & a^{\prime} & a^{\prime} & a^{\prime} & \left\{e, b^{\prime}\right\} \cup P_{1} & \left\{a^{\prime}, b^{\prime}\right\} \\ b^{\prime} & b^{\prime} & b^{\prime} & b^{\prime} & b^{\prime} & \left\{a^{\prime}, b^{\prime}\right\} & \left\{e, a^{\prime}\right\} \cup P_{1}\end{array}$.

For $n=4, m=3, d\left(\mathcal{P}_{1}\right)=\frac{5}{8}, d\left(\mathcal{P}_{2}\right)=1$ it is obatined:

$$
d\left(\mathcal{P}_{1}\left[\mathcal{P}_{2}\right]\right)=\frac{4^{2} \cdot \frac{5}{8}+3^{2} \cdot 1+2(4-1)(3-1)-1}{(4+3-1)^{2}}=\frac{5}{6} .
$$

We notice that $\frac{5}{6}>\frac{5}{8}$, so the result from group theory dosen't holds in polygroup theory.

Remark 17. If $\mathcal{A}$ şi $\mathcal{B}$ are two commutative polygroups, then $d(\mathcal{A})=d(\mathcal{B})=1$ and

$$
d(\mathcal{A}[\mathcal{B}])=\frac{n^{2}+m^{2}+2(n-1)(m-1)-1}{(n+m-1)^{2}}=1 .
$$

So, $\mathcal{A}[\mathcal{B}]$ it is a commutative polygroup.

Remark 18. The polygroup $P_{G}=G \cup\{a\}, a \notin G$, could be written as a extension of polygroup $\mathcal{A}=\left\langle G, \cdot, e,^{-1}\right\rangle$ by polygroup $\mathcal{B}=\left\langle B, \cdot, e,^{-1}\right\rangle$, where $B=\{e, a\}, a \notin G$ and $" . "$ from $\mathcal{B}$ has the form:

the formula $(9)$ for $d(\mathcal{A})=d(G), m=2$ and $d(\mathcal{B})=1$, we obtain

\section{Applying}

$$
d(\mathcal{A}[\mathcal{B}])=\frac{n^{2} d(G)+2^{2}+2(n-1)-1}{(n+2-1)^{2}}=\frac{n^{2} d(G)+2 n+1}{(n+1)^{2}}=d\left(P_{G}\right) .
$$

\section{Remark 19.}

$$
\begin{aligned}
& \lim _{n \rightarrow \infty} \frac{n^{2} d(\mathcal{A})+m^{2} d(\mathcal{B})+2(n-1)(m-1)-1}{(n+m-1)^{2}}=d(\mathcal{A}) ; \\
& \lim _{m \rightarrow \infty} \frac{n^{2} d(\mathcal{A})+m^{2} d(\mathcal{B})+2(n-1)(m-1)-1}{(n+m-1)^{2}}=d(\mathcal{B}) .
\end{aligned}
$$

We determine a boundaries for the extension $\mathcal{A}[\mathcal{B}]$, in the following. 
Proposition 20. $\min \{d(\mathcal{A}), d(\mathcal{B})\} \leq d(\mathcal{A}[\mathcal{B}]) \leq \frac{1+\max \{d(\mathcal{A}), d(\mathcal{B})\}}{2}$.

Proof. Let us suppose that $d(\mathcal{A}) \leq d(\mathcal{B})$. The other case is treated in a similar way.

$$
d(\mathcal{A}[\mathcal{B}]) \geq \frac{n^{2} d(\mathcal{A})+m^{2} d(\mathcal{A})+2(n-1)(m-1)-1}{(n+m-1)^{2}} .
$$

Equivalent with

$$
(1-d(\mathcal{A}))(2 n m+2 n+2 m+1) \geq 0 .
$$

Which is true, because $d(\mathcal{A}) \in(0,1]$.

The next inequality becomes

$$
\begin{aligned}
d(\mathcal{A}[\mathcal{B}]) & \leq \frac{d(\mathcal{B})\left(n^{2}+m^{2}\right)+2(n-1)(m-1)-1}{(n+m-1)^{2}} \\
& =\frac{\left(n^{2}+m^{2}\right)(d(\mathcal{B})-1)}{(n+m-1)^{2}}+1 .
\end{aligned}
$$

But,

$$
\begin{aligned}
\frac{\left(n^{2}+m^{2}\right)(d(\mathcal{B})-1)}{(n+m-1)^{2}}+1 & \leq \frac{1+d(\mathcal{B})}{2} \Leftrightarrow \\
(d(\mathcal{B})-1)\left(\frac{n^{2}+m^{2}}{(n+m-1)^{2}}-\frac{1}{2}\right) & \leq 0,
\end{aligned}
$$

which is true. In conclusion,

$$
\min \{d(\mathcal{A}), d(\mathcal{B})\} \leq d(\mathcal{A}[\mathcal{B}]) \leq \frac{1+\max \{d(\mathcal{A}), d(\mathcal{B})\}}{2} .
$$

\section{On nilpotencity of $\mathcal{A}[\mathcal{B}]$}

In this section, we propose to prove that if $\mathcal{A}$ and $\mathcal{B}$ are two nilpotent polygroups, then the extension of polygropus by polygroups, $\mathcal{A}[\mathcal{B}]$ is also a nilpotent polygroup. To prove this, we need some notions which appears in the book of B. Davvaz, [4].

Definition 21. A polygroup $\left\langle P, \cdot, e,,^{-1}>\right.$ is said to be nilpotent, if $l_{n}(P) \subseteq$ $\omega_{P}$ or equivalent $l_{n}(P) \cdot \omega_{P}=\omega_{P}$, for some integer $n$, where $l_{0}(P) \cdot \omega_{P}=\omega_{P}$ and

$l_{k+1}(P)=<\left\{h \in P \mid x \cdot y \cap h \cdot y \cdot x \neq \varnothing\right.$, such that $x \in l_{k}(P)$ and $\left.y \in P\right\}>$. 
The smallest integer $c$ such that $l_{c}(P) \cdot \omega_{P}=\omega_{P}$ is called the nilpotencity class or for simplicity the class of $P$.

Notice that $P=l_{0}(P) \supseteq l_{1}(P) \supseteq l_{2}(P) \supseteq$...that is $\left\{l_{k}(P)\right\}_{k \geq 0}$ is a decreasing sequence which we call it generalized descending central series.

Forwards, we find a connection between the heart of polygroups $\mathcal{A}, \mathcal{B}$ and $\mathcal{A}[\mathcal{B}]$.

Proposition 22. Let $\mathcal{A}[\mathcal{B}]=<M, *, e,{ }^{I}>$ be the extension of polygroups $\mathcal{A}$ by $\mathcal{B}$, where $M=A \cup B, A \cap B=\{e\}$. The next relation, hold on

$$
\omega_{\mathcal{A}} \cup \omega_{\mathcal{B}} \subseteq \omega_{\mathcal{A}[\mathcal{B}]} .
$$

Proof. Note that the neutral element $e$, is the same in all polygroups, $\mathcal{A}, \mathcal{B}$ and $\mathcal{A}[\mathcal{B}]$.

Let be $x \in \omega_{\mathcal{A}}$, follows that $x \beta^{*} e$, so there is $n \in \mathbb{N}, a_{1}, a_{2}, \ldots, a_{n} \in \mathcal{A}$ such that

$$
\{x, e\} \subseteq \prod_{i=1}^{n} a_{i} .
$$

Using the relation (7), it is observe that $x * y=x \cdot y$, for all $x, y \in \mathcal{A}$. So, the relation (12) could be written thus:

There is $n \in \mathbb{N}, a_{1}, a_{2}, \ldots, a_{n} \in \mathcal{A}[\mathcal{B}]$, such that $\{x, e\} \subseteq a_{1} * a_{2} * \ldots * a_{n}$ which implies $x \in \omega_{\mathcal{A}[\mathcal{B}]}$.

Now, if $x \in \omega_{\mathcal{B}}$, follows that $x \beta^{*} e$, so there is $m \in \mathbb{N}, b_{1}, b_{2}, \ldots, b_{m} \in \mathcal{B}$ such that

$$
\{x, e\} \subseteq \prod_{i=1}^{n} b_{i} \subseteq b_{1} * b_{2} * \ldots * b_{n}
$$

if and only if $b_{i} \neq b_{j}^{-1}, \forall i, j \in \overline{1, n}$, so follows that $x \in \omega_{\mathcal{A}[\mathcal{B}]}$. If exists $i, j$ such that $b_{i}=b_{j}^{-1}, \prod_{i=1}^{n} b_{i} \subseteq b_{1} \cdot b_{2} \cdot \ldots\left(b_{i} \cdot b_{j} \cup A\right) \cdot \ldots \cdot b_{n}$, so $x \in \omega_{\mathcal{A}[\mathcal{B}]}$. In conclusion $\omega_{\mathcal{A}} \cup \omega_{\mathcal{B}} \subseteq \omega_{\mathcal{A}[\mathcal{B}]}$.

Proposition 23. Let $\mathcal{A}=\left\langle A, \cdot, e,^{-1}\right\rangle, \mathcal{B}=\left\langle B, \cdot, e,^{-1}\right\rangle$ be two polygroup. If $\mathcal{A}[\mathcal{B}]=<M, *, e,{ }^{I}>$ is the extension of polygroups $\mathcal{A}$ by $\mathcal{B}$, where $M=$ $A \cup B, A \cap B=\{e\}$, then

$$
l_{k}(\mathcal{A}[\mathcal{B}])=l_{k}(\mathcal{A}) \cup l_{k}(\mathcal{B}) .
$$

Proof. We do the proof

$$
l_{k}(\mathcal{A}) \cup l_{k}(\mathcal{B}) \subseteq l_{k}(\mathcal{A}[\mathcal{B}])
$$


by induction on $k$. For $k=0, A \cup B \subseteq A \cup B$, it is true. Now, suppose that $a \in l_{k+1}(\mathcal{A})$, so exists $x \in l_{k}(\mathcal{A}), y \in \mathcal{A}$, such that

$$
x \cdot y \cap a \cdot y \cdot x \neq \emptyset \text {. }
$$

Using the hypothesis induction, follows that $x \in l_{k}(\mathcal{A}[\mathcal{B}])$.

So, $a \in A \subset A \cup B, x \in l_{k}(\mathcal{A}[\mathcal{B}]), y \in A \cup B$ and

$$
x * y \cap a * y * x \neq \emptyset .
$$

In conclusion, $a \in l_{k+1}(\mathcal{A}[\mathcal{B}])$. If, $a \in l_{k+1}(\mathcal{B})$, exists $x \in l_{k}(\mathcal{B}), y \in \mathcal{B}$, such that $x \cdot y \cap a \cdot y \cdot x \neq \emptyset$. In a similar way, using the hypothesis induction, follows that $x \in l_{k}(\mathcal{A}[\mathcal{B}])$. So, we have two cases:

If, $y \neq x^{-1}$ and $y \neq a^{-1}$, the condition $x \cdot y \cap a \cdot y \cdot x \neq \emptyset$ becomes

$$
x * y \cap a * y * x \neq \emptyset,
$$

where $x \in l_{k}(\mathcal{A}[\mathcal{B}]), a \in A \cup B, y \in A \cup B$, follows that $a \in l_{k+1}(\mathcal{A}[\mathcal{B}])$.

If $y=x^{-1}$ and $y \neq a^{-1}, x * y \cap a * y * x \neq \emptyset$ is equivalent with

$$
(x \cdot y \cup A) \cap(\underset{c \in C}{\cup} a \cdot c) \neq \emptyset,
$$

where $C=y \cdot x \cup A$, because $x \cdot y \cap a \cdot y \cdot x \neq \emptyset$. So, $a \in l_{k+1}(\mathcal{A}[\mathcal{B}])$. The other cases are treated in a similar way.

Now, like above, using the induction method, we do the proof

$$
l_{k}(\mathcal{A}[\mathcal{B}]) \subseteq l_{k}(\mathcal{A}) \cup l_{k}(\mathcal{B}) .
$$

For $k=0, A \cup B \subseteq A \cup B$. If $a \in l_{k+1}(\mathcal{A}[\mathcal{B}])$, then $a \in A \cup B$ and exists $x \in l_{k}(\mathcal{A}[\mathcal{B}]), y \in A \cup B$ such that

$$
x * y \cap a * y * x \neq \emptyset .
$$

Using the hypothesis induction, follows that $x \in l_{k}(\mathcal{A})$ or $x \in l_{k}(\mathcal{B})$.

If $a \in A$, we choose $x \in l_{k}(\mathcal{A})$ and $y \in A$ such that the condition (15) becomes $x \cdot y \cap a \cdot y \cdot x \neq \emptyset$, results $a \in l_{k+1}(\mathcal{A})$.

If $a \in B$ we choose $x \in l_{k}(\mathcal{B})$ and $y \in B$, such that the condition (15) becomes

$$
\left\{\begin{array}{lr}
x \cdot y \cap a \cdot y \cdot x \neq \emptyset, & y \neq a^{-1} \neq x^{-1} \\
(x \cdot y \cup A) \cap(\underset{c \in C}{\cup} a \cdot c) \neq \emptyset, & y=x^{-1} \\
x \cdot y \cap(\underset{d \in D}{\cup} d \cdot x) \neq \emptyset, & y=a^{-1}
\end{array}\right.
$$

where $D=a \cdot y \cup A$. From the relations given by (17), it is obtained that $x \cdot y \cap a \cdot y \cdot x \neq \emptyset$. 
Proposition 24. If $\mathcal{A}$ and $\mathcal{B}$ are nilpotent polygroups, then the extension of polygroups, $\mathcal{A}[\mathcal{B}]$ is also a nilpotent polygroup.

Proof. $\mathcal{A}$ is a nilpotent polygroups, so there exists $k_{1} \in \mathbb{N}^{*}$ such that $l_{k_{1}}(\mathcal{A}) \subseteq$ $\omega_{\mathcal{A}} \cdot \mathcal{B}$ is a nilpotent polygroups, so there exists $k_{2} \in \mathbb{N}^{*}$ such that $l_{k_{2}}(\mathcal{B}) \subseteq$ $\omega_{\mathcal{B}}$.

$$
l_{k_{1}}(\mathcal{A}) \cup l_{k_{2}}(\mathcal{B}) \subseteq \omega_{\mathcal{A}} \cup \omega_{\mathcal{B}} \subseteq \omega_{\mathcal{A}[\mathcal{B}]}
$$

Let $k=\max \left(k_{1}, k_{2}\right)$ and $\left\{l_{k}(P)\right\}_{k \geq 0}$ is a decreasing sequence. We have $l_{k}(\mathcal{A}) \subseteq l_{k_{1}}(\mathcal{A})$ and $l_{k}(\mathcal{B}) \subseteq l_{k_{2}}(\mathcal{B})$. Using the Proposition 23, follows that

$$
l_{k}(\mathcal{A}[\mathcal{B}]) \subseteq \omega_{\mathcal{A}[\mathcal{B}]} .
$$

So, $\mathcal{A}[\mathcal{B}]$ is a nilpotent polygroup.

\section{References}

[1] Ana Castelaz-Commutativity degree of finite groups, A Thesis Submitted to the Graduade Faculty of Wake Forest University, in Partial Fulfillment of the Requirements for the degree of Masters of Arts in the Departament of Mathematics, North Carolina, May (2010).

[2] Piergiulio Corsini- Introducere in teoria hipergrupurilor, Ed. Universităţii Alexandru Ioan Cuza, Iaşi, (1998).

[3] Piergiulio Corsini, Violeta Leoreanu- Applications of hyperstructures theory, Kluwer Academic Publishers Boston/Dordrechtt/ London.

[4] Bijan Davvaz- Polygroup Theory and Related Systems, World Scientific Publishing.

[5] Paul Erdős, Pál Turán- On Some Problems of a Statistical Group Theory , Acta Mathematica Academiae Scientiarum Hungaricae Tomus 19 (3-4), pp.413-435, (1968).

[6] W.H. Gustafson - What is the probability that two group elements commute?, Am. Math. Mon, 80(9):1031 - 1034, (1974).

[7] Azam Hokmabadi, Fahimeh Mohammadzadeh and Elaheh Mohammadzade The commutativity degree of a polygroup, The $6^{\text {th }}$ International Group Theory Conference, 12-13 March 2014, Golestan University, Gorgan, Iran, 88-91.

[8] Li Hongxing, Duan Qinzi and Wang Peizhuang - Hypergroup (I), Busefal, Vol.23, (1985). 
[9] Desmond MacHale - How commutative can a non-commutative group be?, The Mathematical Gazette, Vol. 58, No. 405, pp. 199 - 202, (1974).

[10] G. Miller-Relative number of non-invariant operators in a group,Prco. Nat. Acad. Sci, USA, 30(2):25 - 28, (1944).

[11] Marius Tărnăuceanu - Subgroup commutativity degrees of finite groups, Journal of Algebra, 337, pp. 363 - 368, (2011).

Andromeda Cristina Sonea,

Department of Mathematics,

Alexandru Ioan Cuza, University of Iasi,

Bdul Carol I, 700506, Iasi, Romania.

Email: soneaandromeda@yahoo.com 